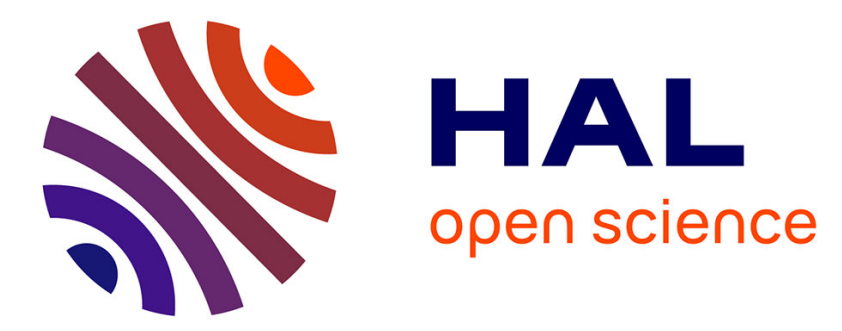

\title{
Physiological Musculoskeletal Model Identification for the Lower Limbs Control of Paraplegic Under Implanted FES
}

\author{
Mourad Benoussaad, David Guiraud, Philippe Poignet
}

\section{To cite this version:}

Mourad Benoussaad, David Guiraud, Philippe Poignet. Physiological Musculoskeletal Model Identification for the Lower Limbs Control of Paraplegic Under Implanted FES. IROS'09: International Conference on Intelligent RObots and Systems, Oct 2009, St. Louis, MO, United States. pp.3549-3554, 10.1109/IROS.2009.5354348 . lirmm-00413447

\section{HAL Id: lirmm-00413447 https://hal-lirmm.ccsd.cnrs.fr/lirmm-00413447}

Submitted on 4 Dec 2009

HAL is a multi-disciplinary open access archive for the deposit and dissemination of scientific research documents, whether they are published or not. The documents may come from teaching and research institutions in France or abroad, or from public or private research centers.
L'archive ouverte pluridisciplinaire HAL, est destinée au dépôt et à la diffusion de documents scientifiques de niveau recherche, publiés ou non, émanant des établissements d'enseignement et de recherche français ou étrangers, des laboratoires publics ou privés. 


\title{
Physiological Musculoskeletal Model Identification for the Lower Limbs Control of Paraplegic Under Implanted FES
}

\author{
Mourad BENOUSSAAD, David GUIRAUD and Philippe POIGNET \\ DEMAR team: LIRMM - INRIA / Montpellier II University / CNRS \\ 161 rue Ada 34392 Montpellier Cedex 5 - France \\ \{benoussa,guiraud,poignet\}@ lirmm.fr
}

\begin{abstract}
This paper concerns the whole physiological parameters identification of a musculoskeletal model of a human subject. The patient is equipped with an implanted Functional Electrical Stimulation (FES) system as part of the SUAW's European project [1]. The biomechanical model represents the knee and its associated muscles. The identification protocol is noninvasive and based on the in-vivo experimental data acquisition of a Spinal Cord Injured (SCI) patient. However, the human noninvasive identification poses problems of inaccessibility to some data.

The identification procedure consists of several steps, in order to identify: the anthropometrical parameters, the geometrical parameters, the joint mechanical parameters, the force-length relationship and the recruitment function. Up to now, only the quadriceps muscle is considered with the knee joint in the identification procedure. A cross-validation has been done using data set not used during the identification process. The identified model shows a satisfactory response comparing to the measured knee response, which is obtained by stimulating the quadriceps through the implanted FES system. In this work, knee model-parameters of the implanted subject were identified successfully using the noninvasive identification procedure.
\end{abstract}

\section{INTRODUCTION}

The movement of limbs, in healthy subjects, is performed from Central Nervous System (CNS), which sends neural signals to muscular fibers that produce the required force. This natural control becomes impossible in the case of a subject with a complete spinal cord lesion, which leads to paralysis. Functional Electrical Stimulation (FES) may then be used to substitute the CNS by contracting the skeletal muscles [2]. In addition, FES presents many therapeutic benefits for SCI patients. However, the restoration of human paralyzed muscle-limb involves a complicate control problem due to the high complexity, nonlinearity and the time variation of the fatigued stimulated muscle.

An accurate numerical model of the muscle-limb dynamics is needed for the model-based control technique. Hill [3] described the macroscopic mechanical characteristics of muscle while Huxley [4] detailed its microscopic concepts. An adaptation of these models to FES control was done in [5],[6]. The accuracy of the model implies to identify several parameters using the FES signal as an excitation input. The physiological parameters of isolated animal muscle were successfully identified in isometric condition using the Sigma-Point Kalman Filtering [7].

Identification of the quadriceps-shank dynamics in nonisometric condition was investigated in several works, online
[8],[9] or off-line [10],[11]. An identification in isometric and isotonic conditions was achieved in [12] based on a black-box nonlinear model. In these works surface electrodes are used for the stimulation. As far as we know, there is no work about the identification using another stimulation type, although, the neural or epimysial implanted stimulation allows a high accuracy, high selectivity and the repeatability of the muscle's response. The SUAW project [1] succeeded in the implantation of an advanced neuroprosthetic device on two patients, using either epimysial or neural electrodes. The goal of the work presented in this paper, is the identification of the quadriceps-shank system, stimulated through neural electrodes, of one subject of the SUAW European project [1]. A combination of both isometric and nonisometric identification is applied and the measured kinetic data set is used for a cross-validation. The identified model allows predicting the behavior of the quadriceps-shank system, which is very useful for the synthesis of the optimal stimulation patterns in the movement restoration context [13].

In the next section, the experimental setup and the patient characteristics are introduced; the knee biomechanical model and the physiological muscle model are presented in section III. In section IV, the parameters identification protocol is described. In section $\mathrm{V}$, we present and discuss the results. Section VI presents the conclusion and the perspectives.

\section{EXPERIMENTAL SET-UP}

The subject is 40 years old, $1.75 \mathrm{~m}$ tall and weighs $66 \mathrm{~kg}$. His paraplegia is ASIA A (American Spinal Injury Association) type T8 level. He has an FES system implanted able to stimulate six different muscles per leg to perform all the kinematics needed for balanced standing and walking. It stimulates through either epimysial or neural electrodes: (i) the gluteus maximus, the gluteus medius and the iliacus for hip flexion-extension and stabilization; (ii) the quadriceps and the hamstrings for knee flexion-extension and (iii) the tibialis anterior for foot dorsal flexion.

In this work, only the quadriceps muscle group is stimulated, through a neural electrode, for the knee extension while the other muscles are considered as having a passive effect. In fact, the unstimulated muscles contribute through its parallel element described in the mechanical model of the Hill-Maxwell model (figure 4). All passive effects of muscles are thus usually reported to the joint dynamics. This assumption is often made to separate the passive and active 


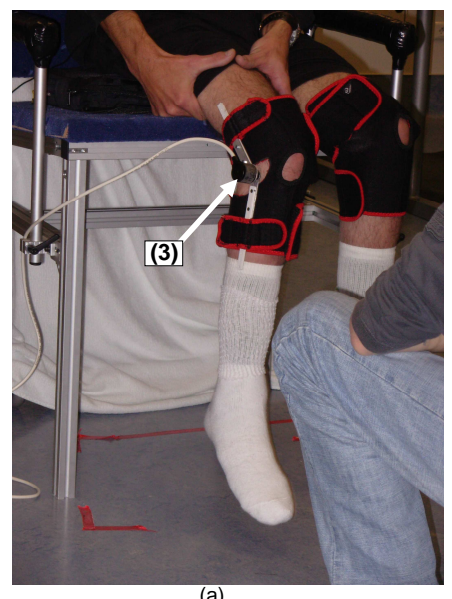

(a)

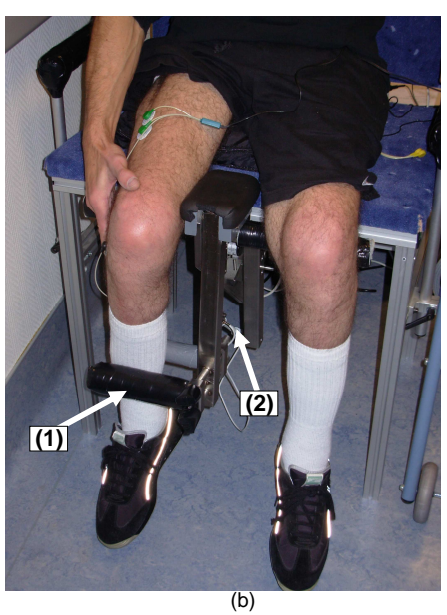

(b)
Fig. 1. The experimental set-up. The subject is seated; the thigh and the trunk were fixed. (a) The shank is free and the angles were measured with an externally mounted electrogoniometer (device 3). (b) The shank is fixed at different positions using the fixation system (device 1) and the torques were recorded through a force sensor (device 2 ).

identification like in [10], where the passive parameters of the joint (including the passive effect of muscles) were assumed independent of the active quadriceps torque. During all experimental procedure, the paraplegic patient is seated on a chair with the thigh and the trunk fixed as shown in figure 1 . The right knee angle was recorded in free shank situation using an electrogoniometer with a $40 \mathrm{~ms}$ sampling period as shown on figure 1.a. In isometric case, the right knee angle was fixed (see figure 1.b), the torques were recorded with a $396 \mu \mathrm{s}$ sampling period using the force sensor of the chair developed in our previous works. The implanted stimulation system [1] is controlled by a laptop and can generate a square stimulation waveform at different frequencies, pulse width and amplitudes.

\section{MODELING}

The biomechanical knee model includes two parts, which are: a) the knee joint model and b) the stimulated muscle model (actuator).

\section{A. Knee joint model}

We consider a 2D biomechanical model in the sagittal plane with one degree of freedom, characterizing the knee joint, controlled by the quadriceps muscle group. Figure 2 represents the joint, where $\theta$ is the knee joint angle, $L_{f}$ the femur length and $L_{o g}$ the distance between the center of mass of the shank and the center of the joint. We model the knee joint as a pulley of radius $r_{1}$ supporting the quadriceps muscles. $\mathbf{F}_{q}$ is the quadriceps force applied on the shank. $M_{s}$ is the shank mass and $g=9.8 \mathrm{~m} / \mathrm{s}^{2}$ is the acceleration of gravity. The rest position is at $\theta=\frac{\pi}{2}$ and the full knee extension is at $\theta=0$. The quadriceps controls the knee joint extension while the gravity force allows the knee flexion. From the illustration 2, the geometrical formulation of quadriceps muscle length is easily given by eq. (1):

$$
L_{q}(\theta)=L_{q_{e x t}}+r_{1} \cdot \theta
$$

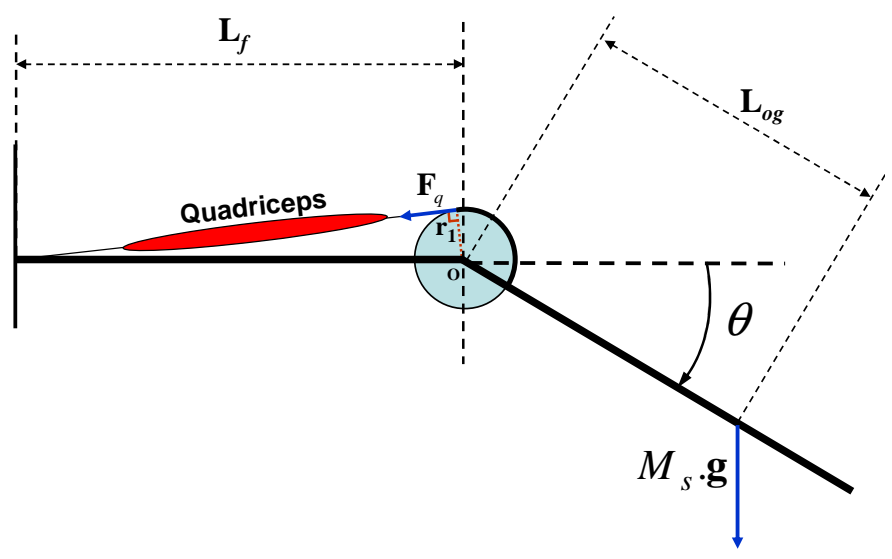

Fig. 2. Knee biomechanical model. The moment arms of muscular force are the pulley radius $r_{1}$. The quadriceps controls the knee joint extension.

Where $L_{q_{\text {ext }}}$ is the quadriceps length at the maximal extension (i.e. $\theta=0^{\circ}$ ).

The dynamic behavior of the joint around the rest position $(\pi / 2)$ is given by the following second order nonlinear equation [14]:

$$
T_{q}=J \cdot \frac{d^{2} \gamma}{d t^{2}}+F_{v} \cdot \frac{d \gamma}{d t}+T_{g}+T_{e}
$$

Where, $\gamma=\frac{\pi}{2}-\theta$ is the knee angles from the vertical in counter-clockwise direction. $T_{q}=F_{q} \cdot r_{1}$ is the active quadriceps torque, $T_{g}=M_{s} \cdot g \cdot L_{o g} \cdot \sin (\gamma)$ is the gravity torque and $T_{e}=K_{e} \cdot \gamma$ is the elastic torque. $F_{v}$ and $K_{e}$ are respectively the viscosity and elasticity coefficients; $J$ is the shank inertia around the center of rotation $O$.

\section{B. Muscle model under FES}

The muscle model used in the following is from the one proposed in [6] simplified considering the muscular fiber fusion assumption as proposed in [15]. One of the main aspects of this model is that its input is the FES signal. The square signal is described by its pulse width $P W$, amplitude $I$ and frequency $f$. The control of one of these parameters leads to the control of the muscular force. The muscle model is composed of two parts (figure 3):

- The activation model: It describes the fiber recruitment function. It represents the relation between the electrical charge applied on the muscle and the ratio of the activated fiber. Its outputs is the recruitment rate $\alpha$. We assume that the stimulation frequency is higher than the fusion frequency of the fibers, which makes a chemical control constant (i.e. $u=1)[15]$. This corresponds to the tetanic contraction, which is obtained here at $31.25 \mathrm{~Hz}$.

- The mechanical muscle model is based on the HillMaxwell structure [3] (figure 4). It includes the contractile element Ec. Es and Ep are serial and parallel elements and represent the passive effect of the tendons. $F_{c}$ and $F_{s}$ are the forces of the contractile and the serial elements respectively and $L_{c}, L_{s}$ are their lengths. As discussed above, the effect of the parallel elements $E p$ is reported to the knee joint as a part of passive effect. 


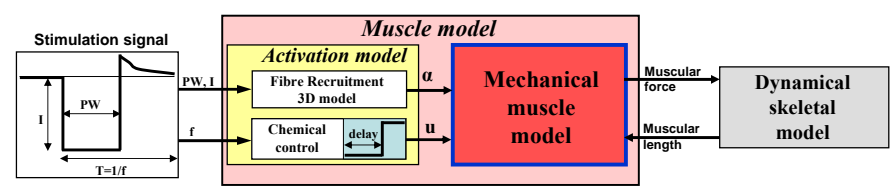

Fig. 3. Complete muscle model [6]. The muscle applies a force on the skeletal system while the joint position has effect back on the muscle length.

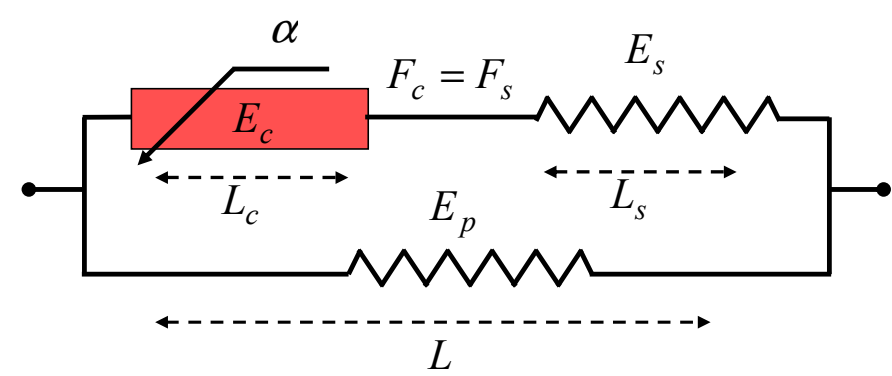

Fig. 4. Controlled mechanical muscle model. $F_{c}$ and $F_{s}$ are the forces of the contractile and the serial elements. The contractile element state is controlled by the recruitment rate $\alpha$.

The dynamical equations, describing the contractile element of the muscle, are based on those presented in [6] and are as follows:

$$
\left\{\begin{array}{l}
\dot{K}_{c}=\left(K_{0}+\frac{s_{v} q F_{0} K_{c}}{1+p K_{c}-s_{v} q F_{c}} K_{c}\right) \alpha-\left(1+\frac{s_{v} q F_{c}+s_{v} a \dot{\varepsilon}}{1+p K_{c}-s_{v} q F_{c}}\right) K_{c} \\
\dot{F}_{c}=\frac{F_{0}}{1+p K_{c}-s_{v} q F_{c}} \alpha+\frac{\left(b K_{c}-s_{v} a F_{c}\right) \dot{\varepsilon}-F_{c}}{1+p K_{c}-s_{v} q F_{c}}
\end{array}\right.
$$

Where, $F_{c}$ and $K_{c}$ are respectively the force and the stiffness of the contractile element and $F_{0}, K_{0}$ are their maximum. $F_{0}$ and $K_{0}$ are related to the muscle length through the forcelength relationship that we will define later. $a, b, p$ and $q$ are constants of the muscle, such as:

$a=\frac{L_{0}}{L_{c 0}}, b=L_{0}, p=\frac{1}{K_{s}}$ and $q=\frac{1}{L_{c 0} K_{s}}$

Where $L_{0}, L_{c 0}$ are the rest lengths of the whole muscle and its contractile element, and $K_{S}$ is the serial element stiffness. $\alpha=f(P W, I)$ is the recruitment rate of muscle, which is a function of the stimulation signal parameters.

$s_{v}$ is equal to $\operatorname{sign}\left(\dot{\varepsilon}_{c}\right)$, where $\varepsilon_{c}$ is the relative deformation of the contractile element. $\varepsilon$ is the relative deformation of the whole muscle.

\section{IDENTIFICATION PROTOCOL}

The identification procedure requires data recorded on a human subject. However, many data or parameters are very difficult or impossible to obtain directly with noninvasive protocols unlike the animal experiments where the muscle can be isolated [7]. Our identification protocol includes five successive parts, which are the identification of:

1) Anthropometrical parameters

2) Geometrical parameters

3) Joint mechanical parameters

4) Force-length relationship

5) Recruitment function
For the whole protocol, the stimulation frequency is fixed at $31.25 \mathrm{~Hz}$, which corresponding to the tetanic contraction and respecting the assumption above.

\section{A. The anthropometrical parameters}

In this method, usually used [11], [9] and well discussed in [14], the mass $M_{s}$, the inertia $J$ and the distance from the knee center to the center of mass $L_{o g}$, were estimated from the mass and length of the whole body through the regression equations [16].

\section{B. The geometrical parameters}

The geometrical estimation provides the value of $L_{q_{\text {ext }}}$ and $r_{1}$ of the equation (1) using the quadriceps length at different samples of knee angle. Quadriceps length is obtained from the Hawkins laws [17]. Linear least square method is applied, through the equation 1 , to obtain $r_{1}, L_{q_{e x t}}$.

\section{The mechanical protocol}

In order to identify the mechanical parameters $F_{v}$ and $K_{e}$ of the equation 2 , the passive pendulum test is usually used. This test is based on the passive oscillation movement of the shank around the rest position without any muscle activation $\left(T_{q}=0\right)$. Assuming small oscillations around the rest position $(\sin (\gamma) \approx \gamma)$, The equation 2 becomes linear as follows:

$$
J \cdot \frac{d^{2} \gamma}{d t^{2}}+F_{v} \cdot \frac{d \gamma}{d t}+K_{e q} \cdot \gamma=0
$$

Where $K_{e q}=M_{s} \cdot g \cdot L_{o g}+K_{e}$ is called a virtual stiffness [14]. During this test, the angles were measured using the electrogoniometer (figure 1.a). These data were filtered using Butterworth filter low-pass with a cutoff frequency of $30 \mathrm{~Hz}$. From the analytical procedure, we obtained the values of the natural frequency $W_{n}=\sqrt{K_{e q} / J}$ and damping ratio $\zeta=$ $F_{v} /\left(2 \sqrt{K_{e q} J}\right)$.

\section{Force-length relationship}

The force-length equation (Eq.5, Eq.6) is a relationship between muscular maximal force/stiffness $\left(F_{0} / K_{0}\right)$ and muscle length, which is related to the knee angle. In isometric condition, we stimulated the quadriceps with the maximal amplitude $I=3 m A$ and a maximal pulse width $P W=600 \mu s$. A shank was fixed at only 5 angles, which corresponds to 5 different muscle lengths, $\left(7^{\circ}, 24^{\circ}, 42^{\circ}, 60^{\circ}, 77^{\circ}\right)$ in order to avoid fatigue. The corresponding maximal torque $\Gamma_{q}$ was measured, filtered and averaged to obtain the corresponding maximal muscular force. From the maximal forces $F_{0}$ and their corresponding muscle lengths estimated using equation 1 , we used the linear least square method to identify the parameter of shape $b_{l}$ from the following exponential equation:

$$
F_{0}\left(L_{q}\right)=F_{\text {max }} \cdot \exp \left[-\left(\frac{L_{q} / L_{o p t}-1}{b_{l}}\right)^{2}\right]
$$

Where, $F_{\max }$ is the maximal force and $L_{o p t}$ its corresponding muscle length. The parameters of equation 6 are the same than those identified previously except the maximal stiffness, which could not be measured through this protocol. Indeed, 


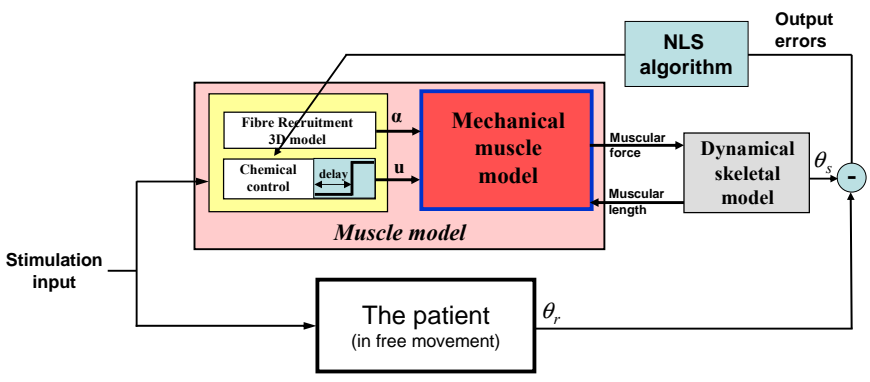

Fig. 5. The recruitment identification principle. We used the nonlinear least square (NLS) to identify the recruitment function parameters, which minimizes the angular joint least square errors.

it involves a fast change of force around a small displacement of muscle length and requires a supplementary device that we did not have. However, we notice a very small sensitivity of the used model to the parameter $K_{\max }$, thus it was taken from the literature and given by $K_{\max }=10^{4}$.

$$
K_{0}\left(L_{q}\right)=K_{\max } \cdot \exp \left[-\left(\frac{L_{q} / L_{o p t}-1}{b_{l}}\right)^{2}\right]
$$

\section{E. Recruitment function}

In this work, the pulse width was fixed to $P W=430 \mu \mathrm{s}$ and only the amplitude of stimulation is changing. Then the recruitment function is a $2 \mathrm{D}$ function as presented below:

$$
\left\{\begin{array}{l}
\alpha(I)=D_{r}\left(\tanh \left(R-C_{r}\right)+\tanh \left(C_{r}\right)\right) \\
\text { with } \\
R=B_{r}\left(1+a_{I} \frac{I}{I_{\max }}\right) I
\end{array}\right.
$$

In this protocol, we used the dynamical movement of the shank. The stimulation-determined patterns were chosen experimentally taking into account the patient safety and avoiding the fatigue. It include the amplitudes between $0 m A$ and $3 m A$, which correspond to the whole range of the recruitment function. The angles were recorded using the electrogoniometer. These input/output data were used to identify the recruitment function parameters $\left(a_{I}, B_{r}, C_{r}, D_{r}\right)$ through a nonlinear least square method as shown on figure 5 .

\section{RESULTS AND DISCUSSION}

The identification parameters are summarized on Table I. The performance of each identification step was quantitatively estimated using RMS error, calculated between predicted and measured data. The RMS error was normalized to the RMS value of the measured data (NRMSE) as presented in [10]. The parameters values obtained from the anthropometrical estimation are the following:

- The shank mass $M_{s}=4.026 \mathrm{~kg}$

- The position of the center of mass $L_{o g}=0.194 \mathrm{~m}$

- The inertia $J=0.343 \mathrm{~kg} \cdot \mathrm{m}^{2}$

The value of shank length and mass seem realistic according to the direct measurement on the subject $(\approx 0.4 m)$ and the body characteristics of the subject. The moment inertia is also realistic comparing to its value obtained from the
TABLE I

PARAMETERS IDENTIFICATION AND CROSS-VALIDATION. NRMSE IS A NORMALIZED RMS ERROR OF IDENTIFICATION (\%). FOR THE ANTHROPOMETRICAL AND GEOMETRICAL PARAMETERS ESTIMATION THERE IS NO EXPERIMENT, THUS THE RMS ERROR COULD NOT BE

\begin{tabular}{|c|c|c|c|}
\hline identification step & NRMSE & Paremeters & Values \\
\hline \multirow{3}{*}{ Anthropometrical } & \multirow{3}{*}{ - } & shank mass $\left(M_{s}\right)$ & $4.026 \mathrm{~kg}$ \\
\hline & & center of mass $\left(L_{o g}\right)$ & $0.194 \mathrm{~m}$ \\
\hline & & shank inertia $(J)$ & $0.343 \mathrm{~kg} \cdot \mathrm{m}^{2}$ \\
\hline \multirow{2}{*}{ Geometrical } & \multirow[t]{2}{*}{ - } & pulley radius $\left(r_{1}\right)$ & $0.048 \mathrm{~m}$ \\
\hline & & $\begin{array}{l}\text { muscle length at } \\
\text { maximal extension } \\
\left(L_{\left.q_{\text {ext }}\right)}\right.\end{array}$ & $0.412 \mathrm{~m}$ \\
\hline \multirow[t]{2}{*}{$\begin{array}{l}\text { Mechanical } \\
\text { of joint }\end{array}$} & \multirow[b]{2}{*}{$25 \%$} & $\begin{array}{l}\text { elasticity coefficient } \\
\left(K_{e}\right)\end{array}$ & $4.441 \mathrm{~N} \cdot \mathrm{m} / \mathrm{rad}$ \\
\hline & & $\begin{array}{l}\text { viscosity coefficient } \\
\left(F_{v}\right)\end{array}$ & $0.201 \mathrm{~N} \cdot \mathrm{m} \cdot \mathrm{s} / \mathrm{rad}$ \\
\hline \multirow{3}{*}{$\begin{array}{l}\text { Force- } \\
\text { length } \\
\text { relationship }\end{array}$} & \multirow{3}{*}{$24 \%$} & shape parameter $\left(b_{l}\right)$ & 0.054 \\
\hline & & $\begin{array}{l}\text { maximal quadriceps } \\
\text { force }\left(F_{\max }\right)\end{array}$ & $230 N$ \\
\hline & & $\begin{array}{l}\text { optimal length of } \\
\text { muscle }\left(L_{o p t}\right)\end{array}$ & $0.455 \mathrm{~m}$ \\
\hline \multirow{4}{*}{$\begin{array}{l}\text { Recruitment } \\
\text { function }\end{array}$} & \multirow{4}{*}{$17.1 \%$} & $a_{I}$ & -0.295 \\
\hline & & $B_{r}$ & 0.593 \\
\hline & & $C_{r}$ & -0.994 \\
\hline & & $D_{r}$ & 4.203 \\
\hline Cross-validation & $16.3 \%$ & - & - \\
\hline
\end{tabular}
EVALUATED.

parallel axis theorem, as shown in [14]. The anthropometrical estimation was often obtained from healthy subjects [16], which can effect the final prediction. However, the limited errors of this identification can be compensated during nonisometric identification, which takes implicitly into account the gravity torque and the inertia of the shank.

The geometrical parameters, which are the pulley radius $r_{1}=0.048 m$ and the quadriceps length at the maximal extension $L_{q_{e x t}}=0.412 \mathrm{~m}$, were obtained. The maximal extension is realistic according to the femur length $L_{f}=0.424 m$, which was obtained from the anthropometrical estimation.

In the mechanical protocol, from the values of the natural frequency $W_{n}=\sqrt{K_{e q} / J}$ and damping ratio $\zeta=$ $F_{v} /\left(2 \sqrt{K_{e q} J}\right)$ of the passive movement, we obtained the virtual stiffness $K_{e q}=W_{n}^{2} \times J=12.115 \mathrm{~N} \cdot \mathrm{m} / \mathrm{rad}$ and the viscous friction $F_{v}=2 \times \zeta \times W_{n} \times J=0.201 \mathrm{~N} \cdot \mathrm{m} \cdot \mathrm{s} / \mathrm{rad}$. Then, we deduce the elasticity coefficient $K_{e}=K_{e q}-m \cdot g . L_{o g}=$ $4.441 \mathrm{~N} \cdot \mathrm{m} / \mathrm{rad}$. Figure 6 illustrates the results, where the measured knee angles and the simulated one appear to be close. However, the normalized RMS error (Table I) seems large because a significant errors appear when the shank stop moving. Indeed, we note that the simulated angles (dotted line) continue to oscillate when the measured one stop moving because we did not include the coulomb friction, as noted in few works [14], which is meaningful only at a very small movement amplitude. The introduction of the coulomb friction improves the knee joint dynamical model but it requires more complex identification procedure.

The shape parameter of the force-length relationship (equations (5)-(6)) is identified as $b_{l}=0.054$. The maxi- 


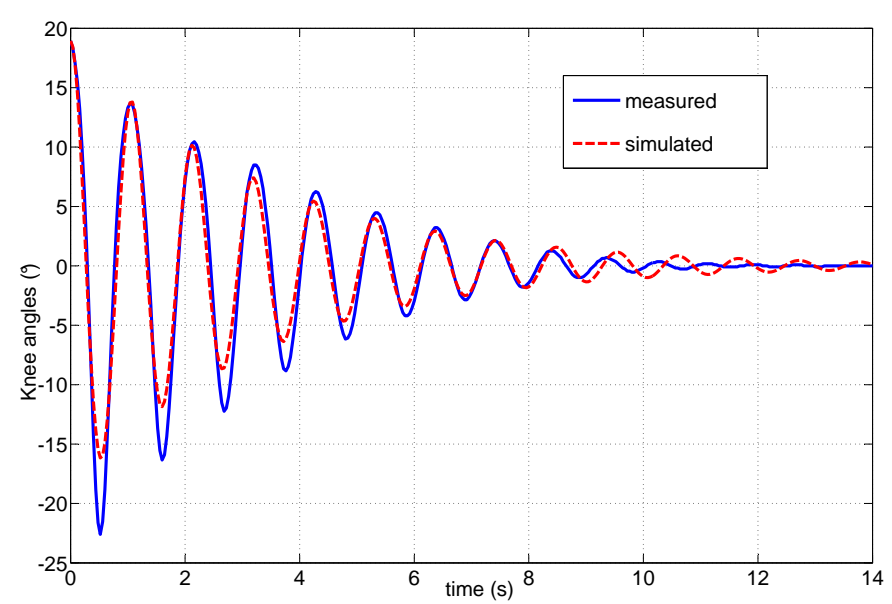

Fig. 6. The oscillation during the passive pendulum. The curve of simulated knee angles (dotted line) obtained from the identified model and the curve of the measured angles recoded from the electrogoniometer appear to be close.

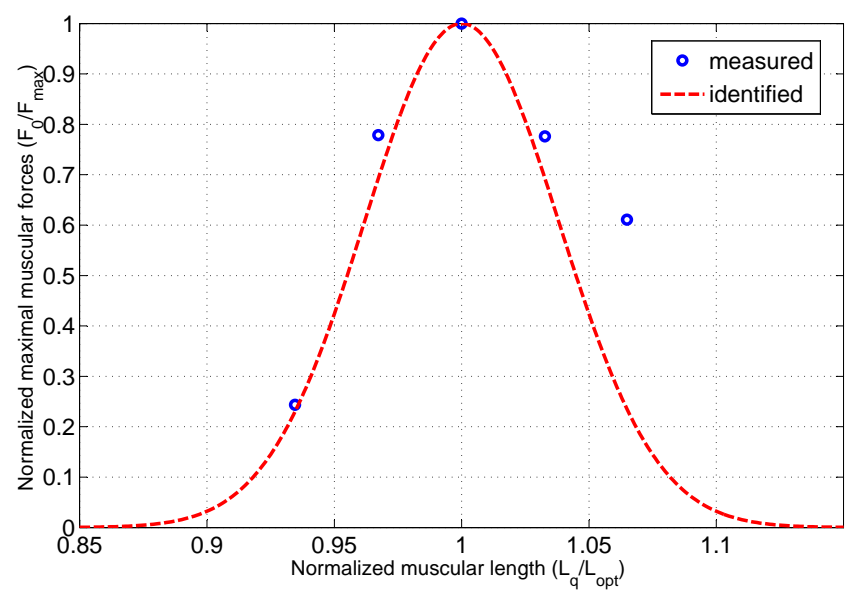

Fig. 7. The measured and identified force-length relationships of the quadriceps. The forces and the muscle lengths were normalized compared with the maximal force $F_{\max }=230 \mathrm{~N}$ and the optimal muscle length $L_{o p t}=$ $0.455 m$.

mal quadriceps force $F_{\max }$ is equal to $230 \mathrm{~N}$ at an optimal muscular length $L_{o p t}=0.455 \mathrm{~m}$. The measured and identified force-length relationship curves are presented on figure 7 . The normalized RMS error is about $24 \%$ (see Table I), which is a relatively large error due to the divergence of the first measured data. In fact, the measured force-length relationship is rarely symmetrical [18] unlike the model.

For the identification of the recruitment function, the excitation stimulation input consist of successive pulses, with an increasing amplitude, as shown on figure 8.a. Figure 8.b illustrates the identification results, where the errors between the measured angles and the simulated one are minimized in terms of least square. The parameters values of this recruitment function was estimated at: $a_{I}=-0.295, B_{r}=$ 0.593, $C_{r}=-0.994, D_{r}=4.203$. The calculated normalized RMS error fitting is around $17 \%$ (see Table I). The error is reduced compared with the errors obtained from the previous
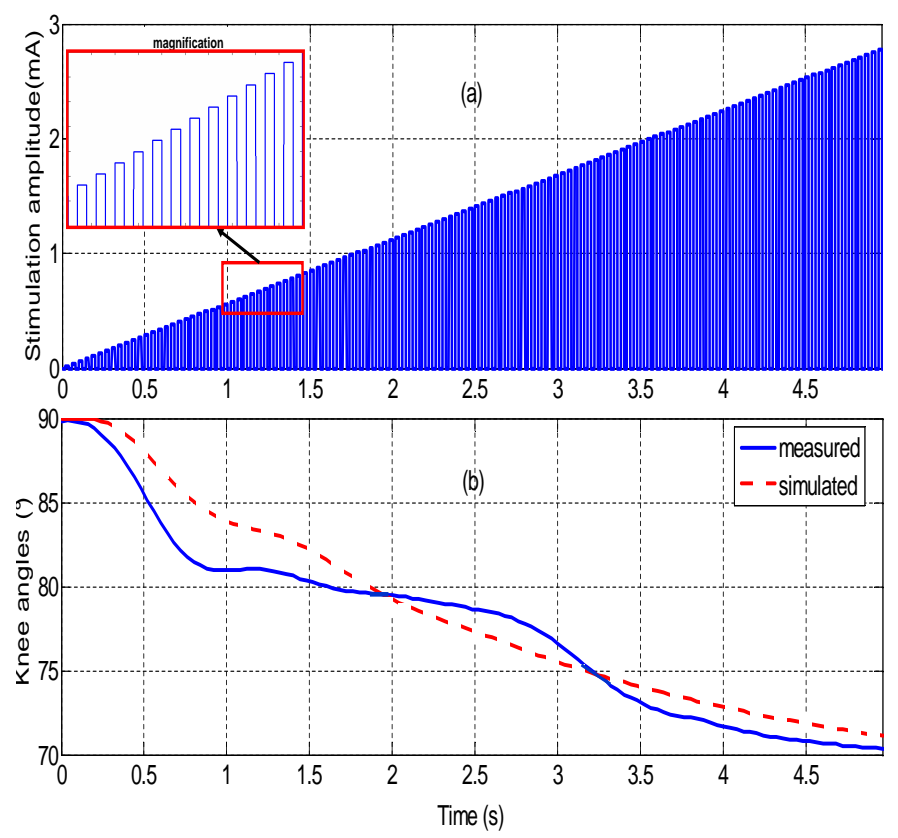

Fig. 8. The stimulation input (a) and the measured/simulated system responses (b) for the identification of recruitment function. The identified parameters are: $a_{I}=-0.295, B_{r}=0.593, C_{r}=-0.994, D_{r}=4.203$.

identifications, which present a significant improvement of the global identified model.

In nonisometric condition, we divided the set of data in two part, the first part is the one used previously for the identification of the recruitment function, and the second part was used for the cross-validation as shown on figure 9. A cross-validation of our model is performed using a stimulation data that have not been used for the identification (second part). Figure 9.a illustrates the amplitudes of this input stimulation, where the frequency and the pulse width are fixed as previously. Figure 9.b shows the simulated knee angles obtained from the identified model (simulated) and the measured response for the same stimulation through the implanted system. The results show a satisfactory agreement between the measured and predicted knee angles considering the quality of the measurements and the difficulties of the noninvasive identification protocol for the implanted subject. The normalized RMS error is about $16.3 \%$ (Table I) and highlights a good agreement between the real and predicted behavior.

All the model parameters described in the identification protocol were identified using a measured data of the subject, while other parameters were taken from the literature. The identification of the missing parameters might improve the quality of the model prediction. It will be investigated in the future works. To understand the influence of each parameter on the identification procedure, the sensitivity study will be inspected in our future works.

\section{CONCLUSIONS AND FUTURE WORKS}

In the current work, the parameters of human subject, implanted in the European SUAW project, were identified 


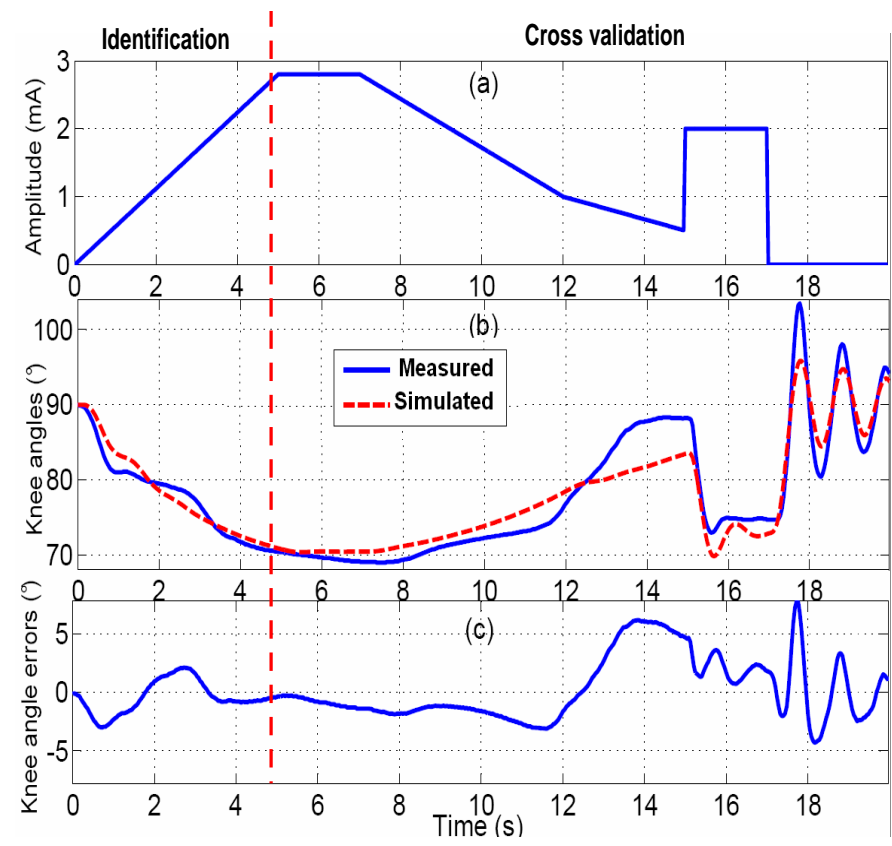

Fig. 9. Data set divided in two parts (for identification and cross-validation). The amplitude of the input stimulation (a). The measured and the identified system responses (b) and the angular errors (c).

using the measured input/output data. The identification procedure was applied to the knee joint and its quadriceps muscle in order to identify:

- The anthropometrical parameters

- The geometrical parameters

- The mechanical joint parameters

- The force-length relationship

- The recruitment function

The quadriceps muscle was stimulated through the implanted stimulator. The identification is based on the measurements of the torque and the knee angle that were made through noninvasive and safe devices.

A cross-validation of the identified model has been performed using a stimulation patterns not used during the identification process. The parameters of the SUAW implanted subject were identified successfully using a noninvasive procedure. The results showed the feasibility of the identification and the validity of the model to predict the implanted subject knee behavior under FES. This prediction is very useful for the synthesis of the appropriate stimulation patterns in the movement restoration context [13]. However, improvements are planned in our future works by using the measurements of the Electromyography (EMG) to obtain the muscular activities. The future works are planned as follows:

1) Synthesize the optimal stimulation patterns to control one knee joint through the quadriceps muscle.

2) Include the identification protocol of the antagonist muscle, which is the hamstring.

3) Apply the same synthesis strategy in order to control the knee joint in single contraction or co-contraction cases.

\section{ACKNOWLEDGMENTS}

Thanks for Christine Azevedo-Coste and Bernard Gilbert for their precious help during the experiments.

\section{REFERENCES}

[1] D. Guiraud, T. Stieglitz, K. P. Koch, J.-L. Divoux, and P. Rabischong, "An implantable neuroprosthesis for standing and walking in paraplegia: 5-year patient follow-up," Journal Of Neural Engineering, vol. 3, pp. 268-275, 2006.

[2] A. Kralj and T. Bajd, Functional Electrical Stimulation: Standing and Walking after Spinal Cord Injury, C. Press, Ed., 1989.

[3] A. Hill, "The heat of shortening and the dynamic constants of muscle," Royal Society of London Proceedings Series B, vol. 126, pp. 136-195, October 1938.

[4] A. F. Huxley, "Muscle structure and theories of contraction," Progress in Biophysics and Biophysical Chemistry, vol. 7, pp. 255-318, 1957.

[5] R. Riener, J. Quintern, E. Psaier, and G. Schmidt., "Physiological based multi-input model of muscle activation," Neuroprosthetics From Basic Research To Clinical Applications, Springer-Verlag, vol. 12, pp. 95-114, 1996.

[6] H. E. Makssoud, D. Guiraud, and P. Poignet, "Mathematical muscle model for functional electrical stimulation control strategies," Proceedings of the 2004 IEEE International Conference on Robotics and Automation, New Orleans, LA, pp. 1282-1287, April 2004.

[7] M. Hayashibe, P. Poignet, D. Guiraud, and H. E. Makssoud, "Nonlinear identification of skeletal muscle dynamics with sigma-point kalman filter for model-based fes," in 2008 IEEE International Conference on Robotics and Automation, Pasadena, CA, USA, May 19-23 2008, pp. 2049-2054.

[8] H. J. Chizeck, S. Chang, R. B. Stein, A. Scheiner, and D. C. Ferencz, "Identification of electrically stimulated quadriceps muscles in paraplegic subjects," IEEE Transactions On Biomedical Engineering, vol. 46, no. 1, pp. 51-61, January 1999.

[9] T. Schauer, N.-O. Negard, F. Previdi, K. Hunt, M. Fraser, E. Ferchland, and J. Raisch, "Online identification and nonlinear control of the electrically stimulated quadriceps muscle," Control Engineering Practice, vol. 13, p. 12071219, October 2004.

[10] D. H. Franken, P. P. Veltink, I. R. Tijsmans, D. H. Nijmeijer, and P. H. Boom, "Identification of passive knee joint and shank dynamics in paraplegics using quadriceps stimulation," IEEE transactions on rehabilitation engineering, vol. 1, no. 3, pp. 154-164, 1993. [Online]. Available: http://doc.utwente.n1/15365/

[11] M. Ferrarin and A. Pedotti, "The relationship between electrical stimulus and joint torque: A dynamic model," IEEE transactions on rehabilitation engineering, vol. 8, no. 3, pp. 342-352, 2000.

[12] F. Previdi, "Identification of black-box nonlinear models for lower limb movement control using functional electrical stimulation," Control Engineering Practice, vol. 10, pp. 91-99(9), January 2002.

[13] M. Benoussaad, P. Poignet, and D. Guiraud, "Optimal functional electrical stimulation patterns synthesis for knee joint control," IEEE/RSJ 2008 International Conference on Intelligent RObots and SystemsNice, France, September, 22-26 2008.

[14] R. B. Stein, E. P. Zehr, M. K. Lebiedowska, D. B. Popović, A. Scheiner, and H. J. Chizeck, "Estimating mechanical parameters of leg segments in individuals with and without physical disabilities,' IEEE Transactions On Rehabilitation Engineering, vol. 4, no. 3, pp. 201-211, September 1996.

[15] S. Mohammed, P. Poignet, and D. Guiraud, "Closed loop nonlinear model predictive control applied on paralyzed muscles to restore lower limbs functions," Proceedings of the 2006 IEEE/RSJ International Conference on Intelligent Robots and Systems, Beijing, China, pp. 259-264, October 9-15 2006.

[16] P. de Leva, "Adjustments to zatsiorsky-seluyanov as segment inertia parameters," Journal of Biomechanics, vol. 29, pp. 1223-1230, 1996.

[17] D. Hawkins and M. Hull, "A method for determining lower extremity muscle-tendon lengths during flexion/extension movements." Journal of Biomechanics, vol. 23, pp. 487-494, 1990.

[18] R. Riener, M. Ferrarin, E. E. Pavan, and C. A. Frigo, "Patient-driven control of FES-supported standing up and sittingdown: experimental results," IEEE Transactions on Rehabilitation Engineering, vol. 8, no. 4, pp. 523-529, Dec. 2000. 